Check for updates

Cite this: RSC Adv., 2018, 8, 3198

Received 2nd December 2017 Accepted 10th January 2018

DOI: $10.1039 / \mathrm{c} 7 \mathrm{ra12980g}$

rsc.li/rsc-advances

\section{Efficient conversion of glucosamine to levulinic acid in a sulfamic acid-catalyzed hydrothermal reaction $\uparrow$}

\author{
Hyo Seon Kim, Sung-Koo Kim and Gwi-Taek Jeong (D) *
}

\begin{abstract}
Glucosamine is a monomer of chitosan, which is a biopolymer derived from the exoskeletons of crustaceans. This work investigated the conversion of glucosamine into the platform chemical levulinic acid (LA), in a sulfamic acid-catalyzed hydrothermal reaction. The optimized results of LA production showed that the conditions of $200{ }^{\circ} \mathrm{C}, 125 \mathrm{~g} \mathrm{~L}^{-1}$ glucosamine, $0.3 \mathrm{M}$ sulfamic acid, and 15 min produced a $33.76 \pm 0.19 \mathrm{~mol} \% \mathrm{LA}$ yield. The same conditions produced only $0.14 \mathrm{~mol} \% 5-\mathrm{HMF}$ yield. These results show that glucosamine is a potential bioresource to produce platform chemicals. Also, in the field of biofuels and chemical synthesis processes, the catalytic system using sulfamic acid is significant.
\end{abstract}

\section{Introduction}

The depletion of fossil resources, increasing energy demand, and changes of global climate are resulting in the active exploration for new energy resources. ${ }^{1-3}$ Bioresources, such as lignocellulosics or macroalgaes, are attractive alternatives to fossil resources, due to their renewability and possible conversion by appropriate technology to various bio-based products. ${ }^{2-7}$ Bio-based products, such as biofuels and platform chemicals, can replace the carbon skeletons derived from fossil resources. ${ }^{2,3,6,7}$

Among the various platform chemicals that can be synthesized from various bioresources, 5-hydroxymethylfurfural (5HMF) offers high potential and versatility for the synthesis of fuels and materials. ${ }^{2,3,7} 5$-HMF can be obtained from various carbohydrates that are derived from sugar, starch, and lignocellulosic biomass. ${ }^{2,3,7}$ Recently, macro-algae and chitin/ chitosan have also been introduced to the production of chemical intermediates. ${ }^{8-15}$ Since 5-HMF has hydroxymethyl and aldehyde groups, it can be converted into several valuable products, which is of high interest for the production of fuels, pharmaceuticals, solvents, resins, fungicides, textiles, and polymer materials by hydrogenation, halogenation, amination, etherification, oxygenation, polymerization, and other chemical reactions..$^{2,3,7,14,15}$

Levulinic acid (LA; 4-oxopentanoic acid), which has two reactive groups, i.e., carbonyl and carboxy groups, has been selected by the U.S. Department of Energy (DOE) as one of the highest valued bio-based chemicals. ${ }^{3}$ These two reactive groups

Department of Biotechnology, Pukyong National University, Busan 48513, Republic of Korea.E-mail: gtjeong@pknu.ac.kr; Fax: +82-51-629-5863; Tel: +82-51-629-5869

$\dagger$ Electronic supplementary information (ESI) available. See DOI: $10.1039 / \mathrm{c} 7 \mathrm{ra12980g}$ can be converted into a wide and valuable range of chemicals. ${ }^{7}$ LA is known as a versatile green chemical, and is now commercially produced by a petrochemical route, using the hydrolysis and hydrogenation of maleic anhydride and furfural alcohol. ${ }^{2,3,6,7}$ Incidentally, this route has some disadvantages, such as environmental problems, and the increase of crude oil price. ${ }^{2,3,6,7}$ To replace the petrochemical route of LA production, renewable bioresources, such as starch-based and lignocellulosic biomass, are being applied to LA production by various chemical processes. ${ }^{2,3,6,7,9,11,14,15}$ Also, LA and its derivatives can be used in the field of pharmaceuticals, fuel extenders, agricultural products, dyes \& coating materials, solvents \& other chemicals, monomers \& specialty polymers, food additives, and others..$^{6,7,11}$

In the present work, glucosamine was employed as a potential bioresource for the production of platform chemicals, namely 5-HMF and levulinic acid. Glucosamine is a major component of chitin/chitosan. Chitin, a copolymer of glucosamine and $N$-acetylglucosamine linked with $\beta-1-4$ bond, is a natural biopolymer that is biologically generated from many aquatic organisms, terrestrial organisms, and some microorganisms. ${ }^{16,17}$ After cellulose, it is the second most abundant nitrogen-containing organic compounds on earth (from $10^{10}$ to $10^{12}$ tons per year). ${ }^{16,18,19}$ Chitosan, polycationic biopolymer, is a deacetylated form of chitin. Nowadays, a large quantity of chitin/chitosan can easily be obtained from by-products as food waste in the shell-crustaceous fish industry. ${ }^{16}$ Because of its properties of nontoxicity, biocompatibility, and biodegradability, ${ }^{16,20,21}$ chitosan has been widely applied in various fields, including cosmetics, medical treatment, biomedicine, food, chemical industries, fibers, sewage treatment, and environmental protection. ${ }^{\mathbf{8 , 1 1 , 1 6 , 2 2}}$

Sulfamic acid has the properties of being non-volatile, odorless, non-corrodible, moderately acidic ( $\left.\mathrm{p} K_{\mathrm{a}}=1.0\right)$, non- 
hygroscopic, and low cost; and also, it is efficient as a green catalyst. ${ }^{23}$ Sulfamic acid has been focused on to provide alternatives to Brønsted- and Lewis acid catalysts, due to its unique catalytic properties and intrinsic zwitterionic nature $\left(\mathrm{H}_{3} \mathrm{~N}^{+} \mathrm{SO}_{3}{ }^{-}\right)$, which occurs by its tautomer structure. ${ }^{23,25}$ In a few years, sulfamic acid has been applied as an efficient acidic catalyst in various acid-catalyzed organic reactions, such as acetalization, esterification, acetylation, Biginelli condensation, Beckmann rearrangement, and Michael reaction..$^{23,26}$

Many works relating to 5-HMF and LA production from various renewable biomasses, such as mono-sugars, starch, and lignocellulosics, have been reported..$^{2,3,6,7,9,11,14,15}$ Moreover, marine macro-algae and chitin/chitosan were recently introduced to produce 5-HMF and LA. ${ }^{8-15}$ Recently, the application of sulfamic acid as catalyst in the 5-HMF and LA production from bamboo fiber was also reported. ${ }^{24}$ However, there has been no study of the conversion of glucosamine using sulfamic acid as a catalyst for platform chemicals, such as 5-HMF and LA.

In this study, the sulfamic acid-catalyzed hydrothermal conversion of glucosamine into the platform chemicals 5-HMF and LA was investigated, and the reaction factors were optimized. Also, the efficiency of sulfamic acid-catalyzed hydrothermal conversion was evaluated with the tool of the combined severity factor (CSF).

\section{Materials and methods}

\section{Materials}

Glucosamine $\mathrm{HCl}$ as the substrate was obtained from Sigma Aldrich Co. Ltd. (USA). The employed sulfamic acid (Ducksan Chemicals, Co. Ltd., Korea) was of reagent grade, and 5-HMF, LA, and all other chemicals were of analytical grade.

\section{Experimental procedure}

The batch experiment for 5-HMF and LA production from glucosamine was performed as follows. In preparatory work, setting amounts of glucosamine and sulfamic acid solution as catalyst were introduced into a $2 \mathrm{~mL}$ glass vial. Prior to sealing, the reactant was sufficiently vortexed for sufficient dissolving and mixing. After these glass vials were sealed, they were inserted into stainless steel reactor for hydrothermal reaction. The reaction was initiated at the time of reaching the setting temperature of the reactor in the oil bath with monitoring and controlling by PID temperature controller. Until reaching the setting temperature, a preheating time of approximately $5 \mathrm{~min}$ was required. Upon completion of the reaction, the reactor was quickly cooled to room temperature by dipping into tap water. For HPLC analysis, the product solution was recovered by centrifugation at $17000 \mathrm{rpm}$ for $10 \mathrm{~min}$, and subsequently filtered using a $0.2 \mu \mathrm{m}$ syringe filter. ${ }^{11}$

\section{Sulfamic acid-catalyzed hydrothermal conversion}

The sulfamic acid-catalyzed conversion of glucosamine was performed to optimize the reaction factors for 5-HMF and LA production: glucosamine concentration $\left(50-150 \mathrm{~g} \mathrm{~L}^{-1}\right)$, reaction temperature $\left(150-220^{\circ} \mathrm{C}\right)$, sulfamic acid concentration $(0.0375-$
$0.5 \mathrm{M})$, and reaction time (0-60 $\mathrm{min})$. Firstly, the effects of biomass concentration were investigated for different glucosamine concentrations in the range of 50-150 $\mathrm{g} \mathrm{L}^{-1}$, under the conditions of $180{ }^{\circ} \mathrm{C}, 0.2 \mathrm{M}$ sulfamic acid, and 30-90 min. Secondly, the effect of reaction temperature was investigated for various temperatures in the range of $150-220{ }^{\circ} \mathrm{C}$, under the conditions of $125 \mathrm{~g} \mathrm{~L}^{-1}$ glucosamine, $0.2 \mathrm{M}$ sulfamic acid, and 30-120 min. Thirdly, the effect of catalyst concentration was investigated for various catalyst concentrations in the range of 0.0375-0.5 M sulfamic acid, under the conditions of $200{ }^{\circ} \mathrm{C}$, $125 \mathrm{~g} \mathrm{~L}^{-1}$ glucosamine, and 15-60 min. Finally, the effect of reaction time in the range of 0-60 min was investigated under the conditions of $200{ }^{\circ} \mathrm{C}, 125 \mathrm{~g} \mathrm{~L}^{-1}$ glucosamine, and $0.3 \mathrm{M}$ sulfamic acid. All of the experiments were performed with two or more repetitions, and the data are presented as the mean $\pm \mathrm{SD}$.

\section{Definition of the combined severity factor}

The effect of the sulfamic acid-catalyzed hydrothermal reaction on 5-HMF and LA production was determined and compared using the combined severity factor (CSF). The CSF indicates the severity of the reaction, which is a function of the reaction conditions of reaction temperature, time, and acidity of solution. ${ }^{14,15,27}$

$$
\mathrm{CSF}=\log \left[t \exp \left(T-T_{\text {ref }}\right) / 14.75\right]-\mathrm{pH}
$$

where, $T(t)$ is the reaction temperature $\left({ }^{\circ} \mathrm{C}\right), T_{\text {ref }}$ is the reference temperature (i.e., 100), $t$ is the reaction time ( $\mathrm{min}$ ), and 14.75 is the fitted value of the arbitrary constant. The $\mathrm{pH}$ of solution was measured at room temperature before the reaction..$^{14,15,27}$

\section{Analysis}

The concentration of glucosamine was analyzed using modified DNS method with spectrophotometry (Spekol 1300, Analytik Jena, Germany) at $540 \mathrm{~nm}$ and glucosamine as standard. The concentrations of 5-HMF and LA were analyzed using an Agilent HPLC system (Agilent 1100, USA) combined with refractive index detector and Aminex-87H column (Bio-Rad, USA). The HPLC operated in the conditions of $65{ }^{\circ} \mathrm{C}$ oven temperature, $5 \mathrm{mM}$ sulfuric acid as mobile phase, and $0.6 \mathrm{~mL} \mathrm{~min}^{-1}$ flow rate. ${ }^{11,14,15}$ For residue (humins) analysis, the product solution was removed by centrifugation at $17000 \mathrm{rpm}$ for $10 \mathrm{~min}$, and subsequently remained residues after washing with distilled water were dried at vacuum oven for $24 \mathrm{~h}$, and the dried solid was considered as insoluble humins. FT-IT spectra of the humins were analyzed from $649-4000 \mathrm{~cm}^{-1}$ at resolution of $4 \mathrm{~cm}^{-1}$ using Fourier Transform Infra Red Spectrometer (Perkin Elmer (U.S.A), Spectrum X).

\section{Determination of the conversion yield}

The yield of glucosamine, 5-HMF, FA and LA was calculated by the following equation: conversion yield $(\mathrm{mol} \%)=$ mole concentration of product $(\mathrm{M}) /$ mole concentration of initial substrate $(\mathrm{M}) \times 100 \%$. Humins yield $(\mathrm{wt} \%)=$ dry weight of residue $(\mathrm{g}) /$ dry weight of initial substrate $(\mathrm{g}) \times 100 \%$. The results were recorded as average $\pm \mathrm{SD}$. 


\section{Results and discussion}

In this study, the sulfamic acid-catalyzed hydrothermal conversion of glucosamine into 5-HMF and LA was investigated. To optimize the production of 5-HMF and LA, the effects of reaction factors of biomass concentration, reaction temperature, catalyst concentration, and reaction time were investigated. Also, the effect of the sulfamic acid-catalyzed hydrothermal reaction was compared using the CSF.

\section{Effect of biomass concentration}

Fig. 1 shows the effect of biomass concentration in the range of 50-150 $\mathrm{g} \mathrm{L}^{-1}$ on 5-HMF and LA yield under the conditions of $180^{\circ} \mathrm{C}, 0.2 \mathrm{M}$ sulfamic acid, and 30-90 min. Fig. 1(A) shows that in the case of 5-HMF production, increasing biomass concentration in the tested ranges sharply decreased the yield of 5HMF. Also, the increase of reaction time caused serious decrease of the 5-HMF yield. The highest 5-HMF yield obtained was $2.76 \pm 0.01 \%$ at $50 \mathrm{~g} \mathrm{~L}^{-1}$ glucosamine. Overall, the yields of 5-HMF were low in the tested biomass ranges. Fig. 1(B) shows that in LA production, increasing biomass concentration in the tested ranges at constant reaction time condition slightly

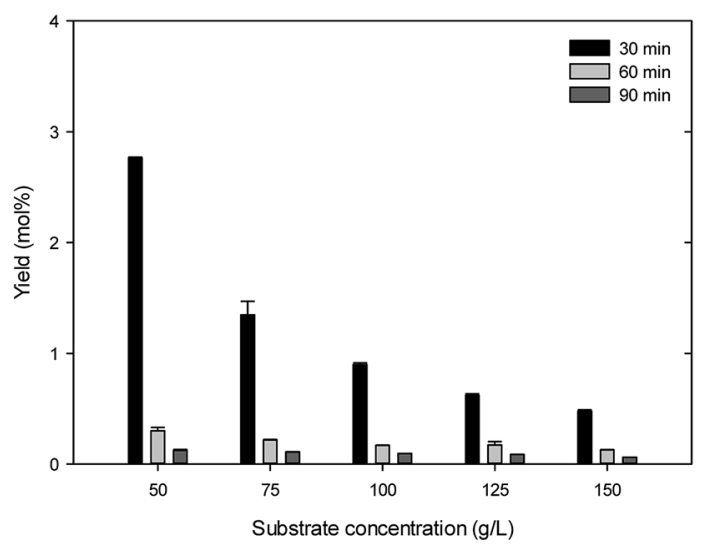

(A) 5 -HMF

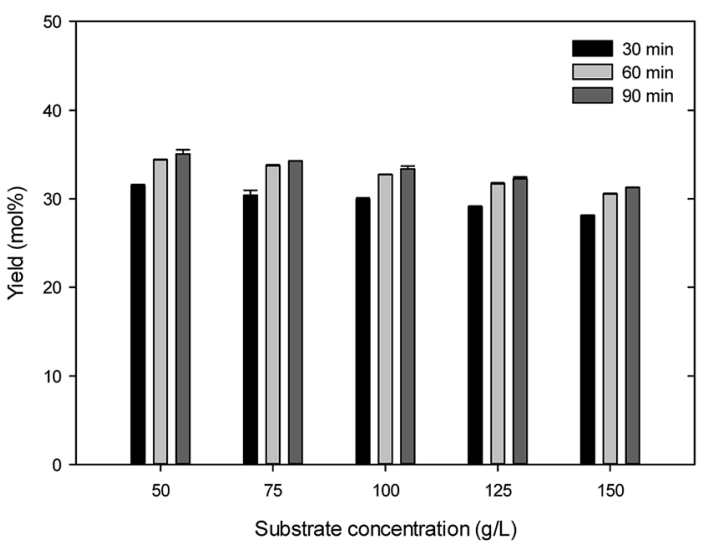

(B) Levulinic acid

Fig. 1 Effect of the biomass concentration on 5-hydroxymethylfurfural and levulinic acid production from glucosamine by sulfamic acid-catalyzed hydrothermal conversion. decreased LA yields. Also, the increase of reaction time produced slight increase of LA yield in the tested range. The highest LA yield achieved was $35.06 \pm 0.47 \%$ at the conditions of $50 \mathrm{~g} \mathrm{~L}^{-1}$ glucosamine and $30 \mathrm{~min}$. Nevertheless, in the conditions of 50-150 $\mathrm{g} \mathrm{L}^{-1}$ range of glucosamine and constant reaction time, the difference of LA yield was little. It is suggested that the limitation of contact between biomass and catalyst resulted in the relatively low LA yield that was achieved at high biomass concentration. ${ }^{\mathbf{1 1}}$ Comparably, increasing the glucosamine concentration within the $50-120 \mathrm{~g} \mathrm{~L}^{-1}$ range in the sulfuric acid-catalyzed hydrothermal reaction slightly decreased the LA yield., ${ }^{\mathbf{4 1 1}}$ Because the production cost is closely related to the initial biomass concentration, further experiment with regard to the LA yield and biomass concentration was conducted under the condition of $125 \mathrm{~g} \mathrm{~L}^{-1}$ glucosamine concentration.

\section{Effect of reaction temperature}

Fig. 2 shows the effect of reaction temperature on the glucosamine conversion into 5-HMF and LA under the conditions of 150-220 ${ }^{\circ} \mathrm{C}, 125 \mathrm{~g} \mathrm{~L}^{-1}$ glucosamine, 0.2 M sulfamic acid, and 30-120 min. Fig. 2(A) shows that in the formation of 5-HMF,

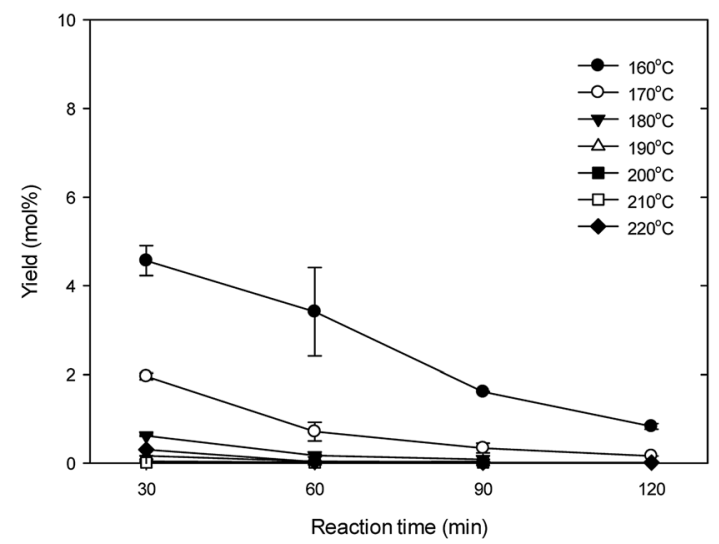

(A) $5-\mathrm{HMF}$

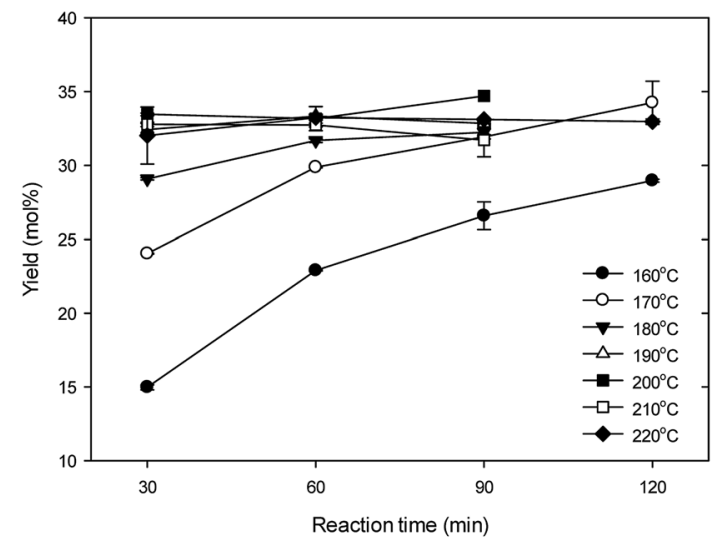

(B) Levulinic acid

Fig. 2 Effect of the reaction temperature on 5-hydroxymethylfurfural and levulinic acid production from glucosamine by sulfamic acidcatalyzed hydrothermal conversion. 
increasing the reaction temperature in the tested ranges sharply decreased the 5-HMF yield. At all reaction temperature ranges, passing reaction time sharply decreased the 5-HMF yield. In particular, the condition of over $180{ }^{\circ} \mathrm{C}$ resulted in the formation of 5-HMF being below 1\%. High 5-HMF yield was obtained at the conditions of low temperature and short reaction time. The highest 5 -HMF yield of $6.36 \pm 0.01 \%$ was obtained at $150{ }^{\circ} \mathrm{C}$ and $30 \mathrm{~min}$. This indicates that under the conditions of higher temperature and longer reaction time, the 5-HMF is easily degraded to LA and FA. ${ }^{\mathbf{8 , 1 1}}$ In comparison, the hydrothermal conversion of $N$-acetyl-D-glucosamine using ionic liquid catalyst under water-DMSO mixture gradually increased the 5-HMF yield, which peaked at $180{ }^{\circ} \mathrm{C}^{13}$ Also, in the sulfamic acidcatalyzed conversion process of bamboo fiber with microwave irradiation in biphasic system, the 5-HMF yield consistently increased, and peaked at $180{ }^{\circ} \mathrm{C}^{24}$

Fig. 2(B) shows that in the formation of LA, increasing reaction temperature linearly increased the LA yields, and then over $200{ }^{\circ} \mathrm{C}$ did not show any significant difference. The highest yield of $34.7 \%$ was achieved at $200{ }^{\circ} \mathrm{C}$ and $90 \mathrm{~min}$. Below $180^{\circ} \mathrm{C}$, passing reaction time linearly increased the LA yield. In particular, the over $200{ }^{\circ} \mathrm{C}$ condition maintained LA yield at similar high values of $32.5-34.7 \%$. The conditions of high temperature and long reaction time formed high LA yield.

Similar to glucose, the results of Fig. 2 suggest that 5-HMF was first formed by glucosamine conversion by the sequential reactions of deamination and dehydration. Subsequently, in the condition of higher temperature, LA and FA was formed by rehydration from 5-HMF. ${ }^{2,9,11}$ In comparison, under the sulfuric acid-catalyzed hydrothermal conversion of glucosamine, LA production was linearly increased within the $160-190{ }^{\circ} \mathrm{C}$ range. ${ }^{11}$ These results might reflect the LA formation from 5HMF, because the activation energy for 5-HMF degradation is lower than that of 5 -HMF formation from sugars. ${ }^{13,28}$ In summary, in this study, higher temperature in the sulfamic acid-catalyzed hydrothermal reaction of glucosamine favored LA production. Therefore, further works were conducted under the reaction temperature of $200{ }^{\circ} \mathrm{C}$.

\section{Effect of catalyst concentration}

Fig. 3 shows the effect of catalyst concentration on the formation of LA in the range of 0.0375-0.5 M sulfamic acid under the conditions of $200{ }^{\circ} \mathrm{C}, 125 \mathrm{~g} \mathrm{~L}^{-1}$ glucosamine, and 15-60 min. In 5 -HMF production, all tested catalyst concentration ranges provided the obtained 5-HMF yield of below $0.17 \%$ (data not shown). In LA production (Fig. 3), increasing of catalyst concentration until $0.3 \mathrm{M}$ slightly increased the LA yield, and then at the $0.5 \mathrm{M}$ catalyst condition was similarly maintained. The highest LA yield of $33 \%$ was obtained under the conditions of $0.3 \mathrm{M}$ sulfamic acid and $60 \mathrm{~min}$. In spite of that, the range of over $0.3 \mathrm{M}$ catalyst concentration produced less difference of LA yield. The increase of access and active sites of catalyst with high sulfamic acid concentration increased LA yield. ${ }^{12,13}$ In comparison, in the sulfuric acid-catalyzed hydrothermal conversion of glucosamine, increasing sulfuric acid concentration within $2-4 \%$ slightly increased the LA yield. ${ }^{\mathbf{1 1}}$ In the $\mathrm{FeCl}_{2}$

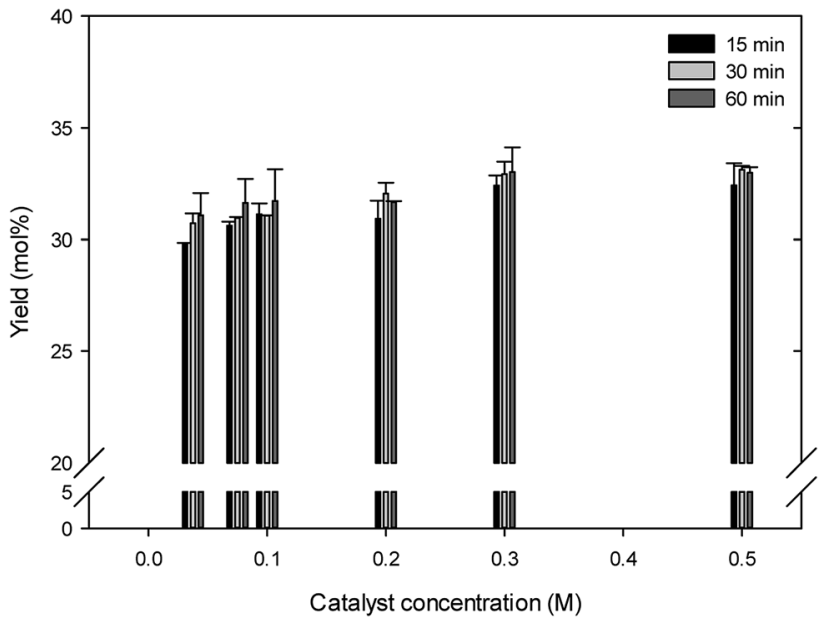

Fig. 3 Effect of the catalyst concentration on levulinic acid production from glucosamine by sulfamic acid-catalyzed hydrothermal conversion.

catalyzed conversion of chitosan and GluNAc, GluNAc showed more significant effect in 5-HMF production than that of chitosan, due to the inhibited access of catalyst to chitosan by the extensive intra- and inter-molecular hydrogen bonds of chitosan. ${ }^{12}$ In summary, considering the catalyst cost and LA yield, further experiments in view of the LA yield and catalyst concentration were therefore carried out under the $0.3 \mathrm{M}$ sulfamic acid condition.

\section{Effect of reaction time}

Fig. 4 shows the effect of the reaction time on LA and other products formation at different reaction times in the range of 0-60 min under the conditions of $200{ }^{\circ} \mathrm{C}, 125 \mathrm{~g} \mathrm{~L}^{-1}$ glucosamine, and $0.3 \mathrm{M}$ sulfamic acid. The conversion of glucosamine sharply increased, and then after $15 \mathrm{~min}$, steadily maintained. The highest glucosamine conversion of $94.5 \%$ was obtained at $60 \mathrm{~min}$. The 5-HMF yield sharply decreased, and then after

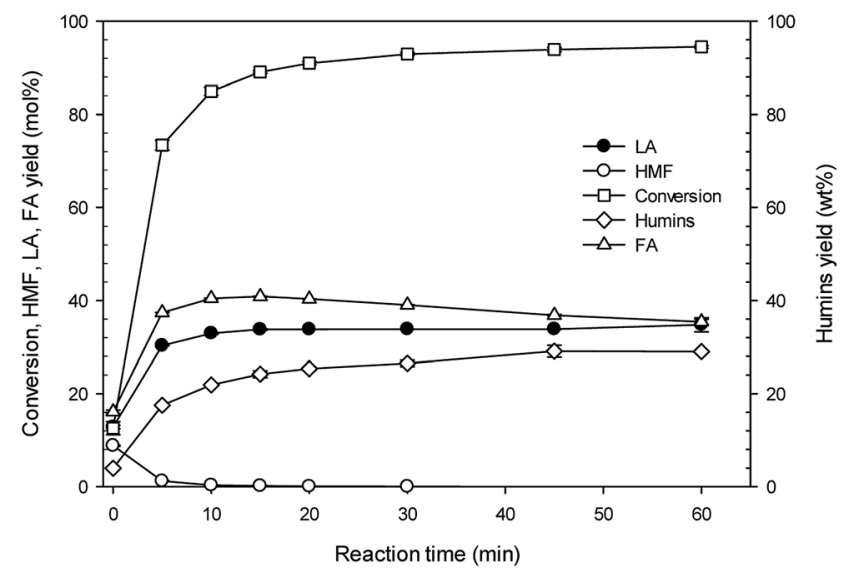

Fig. 4 Effect of the reaction time on 5-hydroxymethylfurfural, levulinic acid, formic acid, humins production from glucosamine by sulfamic acid-catalyzed hydrothermal conversion. 
$10 \mathrm{~min}$, nearly disappeared. The highest 5-HMF yield of $8.82 \pm$ $0.14 \%$ was achieved at $0 \mathrm{~min}$. However, increasing the reaction time until 10 min sharply increased the LA yield. After $20 \mathrm{~min}$, the LA yield was similarly maintained at approximately $33.8 \%$. The pattern of FA formation was similar to LA. At $10 \mathrm{~min}$, FA yield was reached to $40.4 \pm 0.10 \%$. After $30 \mathrm{~min}$, FA yield was decreased. It is indicated that the decomposition of FA to $\mathrm{CO}_{2}$, $\mathrm{H}_{2}$ and $\mathrm{H}_{2} \mathrm{O}$ is occurred under severe harsh conditions. ${ }^{29}$ Moreover, the formation of humins (insoluble dark materials) linearly increased, and then after $15 \mathrm{~min}$, slightly increased with increasing time. It is opposite pattern with LA and FA yield. At $60 \mathrm{~min}$, humins formation was reached to $29.0 \pm 0.05 \%$. The HPLC chromatogram of product analysis was presented in Fig. 1S. $\uparrow$ Glucosamine and sulfamic acid did not determined by our HPLC analysis. FA (13.93 $\mathrm{min}$ ), LA (15.65 $\mathrm{min}$ ) and 5-HMF (29.49 $\mathrm{min}$ ) were detected. Especially, from the experience of HPLC analysis, in the range of 8-11 min of retention time, monomeric sugars was detected (glucose $9.13 \mathrm{~min}$; fructose (or xylose, mannose, galactose) $9.89 \mathrm{~min}$ ). By the way, some unknown peaks were observed at 8-11 min range. Overall, this demonstrates the conversion of glucosamine into LA. Glucosamine is first converted to 5-HMF by deamination and dehydration. Further reaction of rehydration and passing time converts the 5-HMF formed from glucosamine into LA and FA. ${ }^{2,9,11}$ Similarly, 5-HMF shows high yield at the initial period of about $3 \mathrm{~min}$, and then decreases with time passing. ${ }^{11}$ However, in the sulfuric acid-catalyzed hydrothermal reaction of glucosamine, under the $175^{\circ} \mathrm{C}$ and $90 \mathrm{~g} \mathrm{~L}^{-1}$ glucosamine conditions, increasing reaction time linearly increased the LA yield. ${ }^{11}$ From the overall experimental results, in the sulfamic acid-catalyzed hydrothermal reaction of glucosamine, the optimal conditions of LA production are $200{ }^{\circ} \mathrm{C}, 125 \mathrm{~g} \mathrm{~L}^{-1}$ glucosamine, $0.3 \mathrm{M}$ sulfamic acid, and $20 \mathrm{~min}$. These results show the significance and availability of glucosamine and sulfamic acid to the field of platform chemicals production from renewable bioresources.

The several mechanisms of 5-HMF and LA formation by several carbohydrates and catalysts were proposed. ${ }^{6,11,30}$ Several reports have been suggested both cyclic and acyclic reaction routes as the conversion mechanism of glucose. ${ }^{10,30,31}$ Cyclic routes occurred through fructose isomerization..$^{\mathbf{1 0 , 3 0 , 3 1}}$ Glucosamine is the pyranose form, which is similar structure to glucose, with amine group $\left(-\mathrm{NH}_{2}\right) .^{9-11}$ In accordance with previous works, it have been suggested as similar modified route to glucose..$^{32-34}$ Like glucose conversion mechanism, glucosamine (pyranose form with amine group) is first isomerized to the furanose form with amine group. This furanose form was converted into 5-HMF by deamination, dehydration, and keto-enol tautomerization. ${ }^{\mathbf{1 2 , 2 5 , 3 5}}$ Under acidic conditions, the deamination is accelerated.9,10,12,36 Unfortunately, the verification of deamination during glucosamine conversion has been failed by this and other works. ${ }^{\mathbf{9} 12,36}$ Moreover, formed 5HMF is rehydrated to LA and FA under high temperature and high acidic condition. ${ }^{\mathbf{1 1}, \mathbf{1 4}}$

In this study, the glucosamine is converted by sulfamic acid, which owing to its tautomer structure, has dual-catalyst sites of both Brønsted acid and Lewis acid sites. ${ }^{23-25}$ As a previous report, Sun et al. ${ }^{24}$ published that dual-catalyst sites of sulfamic acid might be promote the polymer hydrolysis and subsequent dehydration of glucose to 5-HMF. Although there is no suggestion of detailed mechanism, it is supposed due to the synergetic effect between Lewis acid site of sulfamic acid and the hydroxyl group in cellulose. ${ }^{24}$ In comparison with this work, due to similar structure of glucosamine and glucose, sulfamic acid might have converted the glucosamine more by the synergetic effect of dual-active sites of catalyst. ${ }^{23-25}$ Recently, several works reported that the combining Brønsted and Lewis acids have positive synergistic catalytic effects on the production of 5-HMF and LA from various carbohydrates. ${ }^{36-41}$ Zhang et al. ${ }^{\mathbf{4 0}}$ and Acharjee and Lee ${ }^{\mathbf{4 1}}$ proposed the glucose conversion pathway using dual acid catalysts. They referred that the isomerization of glucose was mostly catalyzed by Lewis acid, whereas, dehydration was catalyzed by Brønsted and Lewis acids. Moreover, the rehydration of 5-HMF to LA and FA was catalyzed by Brønsted acid. ${ }^{\mathbf{4 0 , 4 1}}$ Due to similar structure to glucose, the conversion pathway glucosamine with sulfamic acid was proposed and illustrated as Scheme $1 .^{\mathbf{4 0 , 4 1}}$

We compared and analyzed the structures of glucosamine, sulfamic acid and residues (humins) using Fourier transform infrared (FT-IR) (Fig. 5). The peaks at 3345 and $3275 \mathrm{~cm}^{-1}$ were correspond to $\mathrm{N}-\mathrm{H}$ and $\mathrm{O}-\mathrm{H}$ stretching vibrations. ${ }^{12}$ The peaks at 2997 and $2908 \mathrm{~cm}^{-1}$ were attributed the $\mathrm{C}-\mathrm{H}$ stretching vibrations. The peak at $1545 \mathrm{~cm}^{-1}$ was attributed $-\mathrm{NH}_{2}$ bending vibration. ${ }^{12}$ The band of $1200-1000 \mathrm{~cm}^{-1}$ attributed to stretching of the glycosidic linkage $(\mathrm{C}-\mathrm{O}-\mathrm{C})$ and $\mathrm{C}-\mathrm{OH}$ in the sugar ring. ${ }^{42,43}$ The band of $1350-1000 \mathrm{~cm}^{-1}$ was corresponded amines. The peak at $1274 \mathrm{~cm}^{-1}$ was ascribed sulfonamide. The band of $1054-867 \mathrm{~cm}^{-1}$ was ascribed to pyranose unit of polysaccharide. ${ }^{12}$ Fig. $2 \mathrm{~S} \dagger$ shows the FT-IR spectra of residues

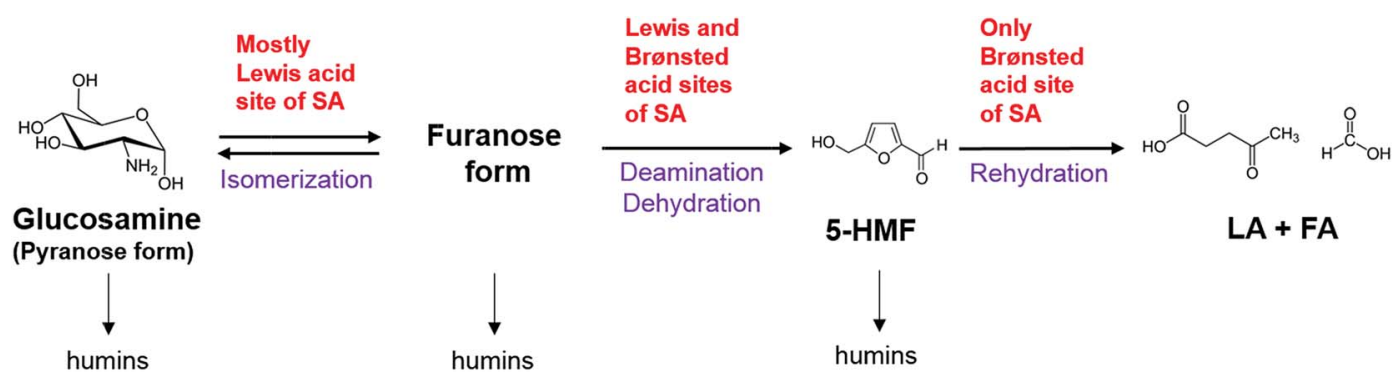

Scheme 1 Overall reaction scheme of sulfamic acid catalyzed production of levulinic acid from glucosamine. ${ }^{40,41}$ 


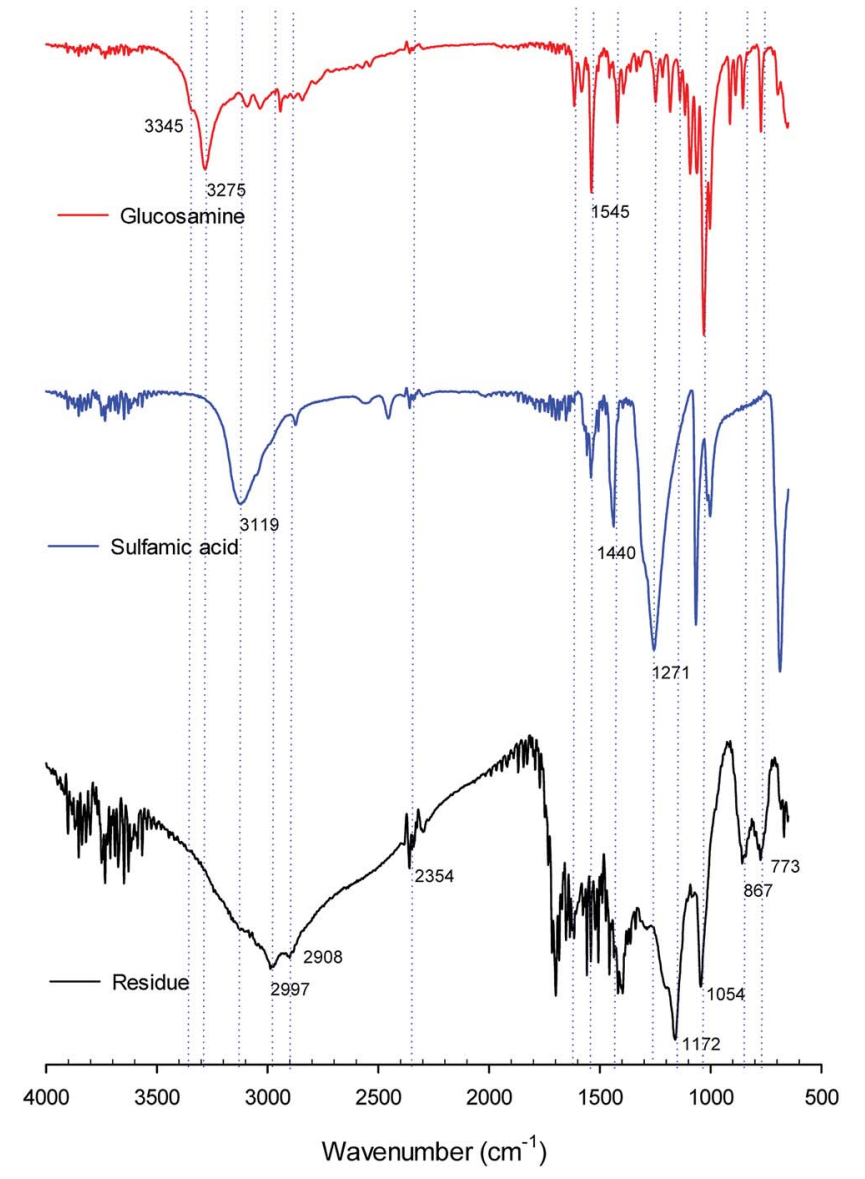

Fig. 5 FT-IR spectra of glucosamine and the residue.

(humins) during conversion reaction of glucosamine with sulfamic acid. The FT-IR spectra of residues showed similar pattern results.

\section{Mass balance of HMF and LA production}

Fig. 6 shows the mass balance diagram of 5-HMF, LA, FA, and insoluble matters (humins) under the conditions of $125 \mathrm{~g} \mathrm{~L}^{-1}$ glucosamine and $0.3 \mathrm{M}$ sulfamic acid at $200{ }^{\circ} \mathrm{C}$ for $30 \mathrm{~min}$. From this condition, the results presented that $125 \mathrm{~g} \mathrm{~L}^{-1}$ glucosamine (92.9\% conversion) could be convert to $22.8 \mathrm{~g} \mathrm{~L}^{-1}$ (33.83 \pm

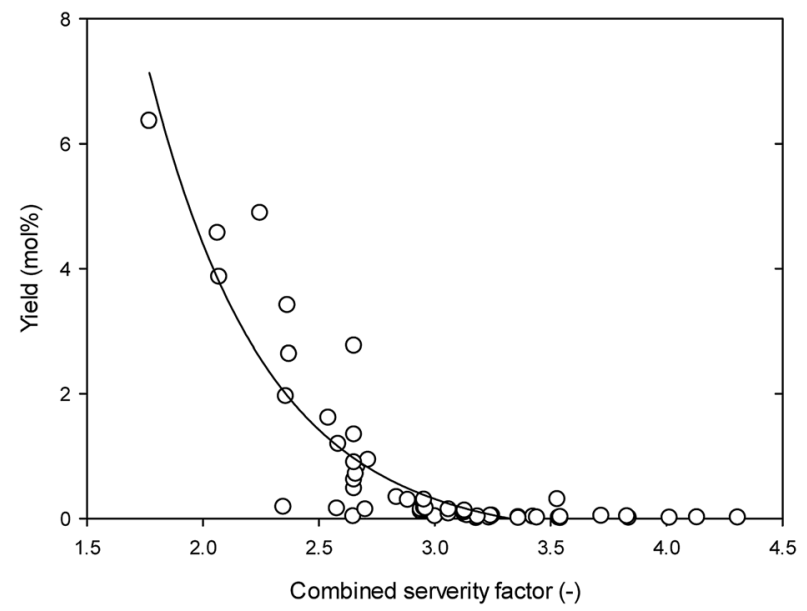

(A) 5 -HMF

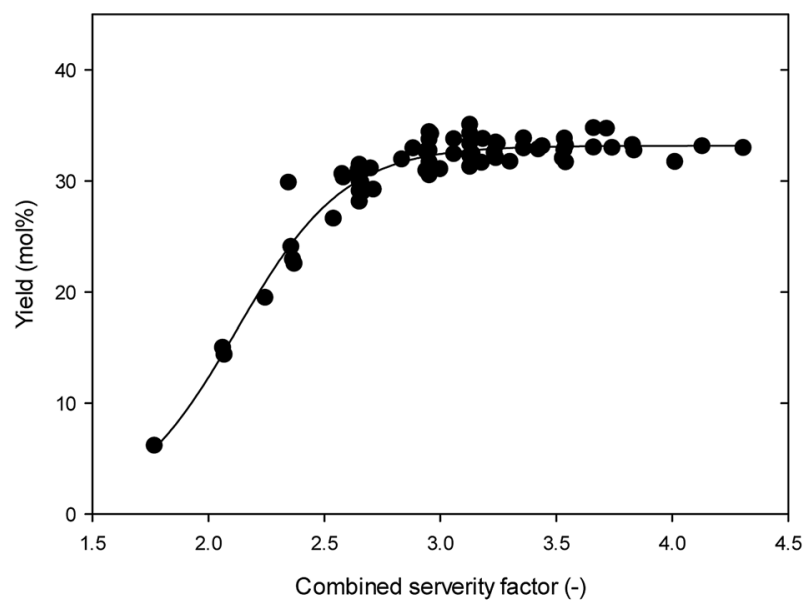

(B) Levulinic acid

Fig. 7 Effect of the combined severity factor on 5-hydroxymethylfurfural and levulinic acid production from glucosamine by sulfamic acid-catalyzed hydrothermal conversion.

$0.03 \mathrm{~mol} \%) \mathrm{LA}, 10.4 \mathrm{~g} \mathrm{~L}^{-1}(39.03 \pm 0.06 \mathrm{~mol} \%) \mathrm{FA}$ and $33.1 \mathrm{~g} \mathrm{~L}^{-1}$ $(26.49 \pm 0.68 \mathrm{wt} \%)$ humins. Especially, 5-HMF detected nearly zero amount under these conditions.

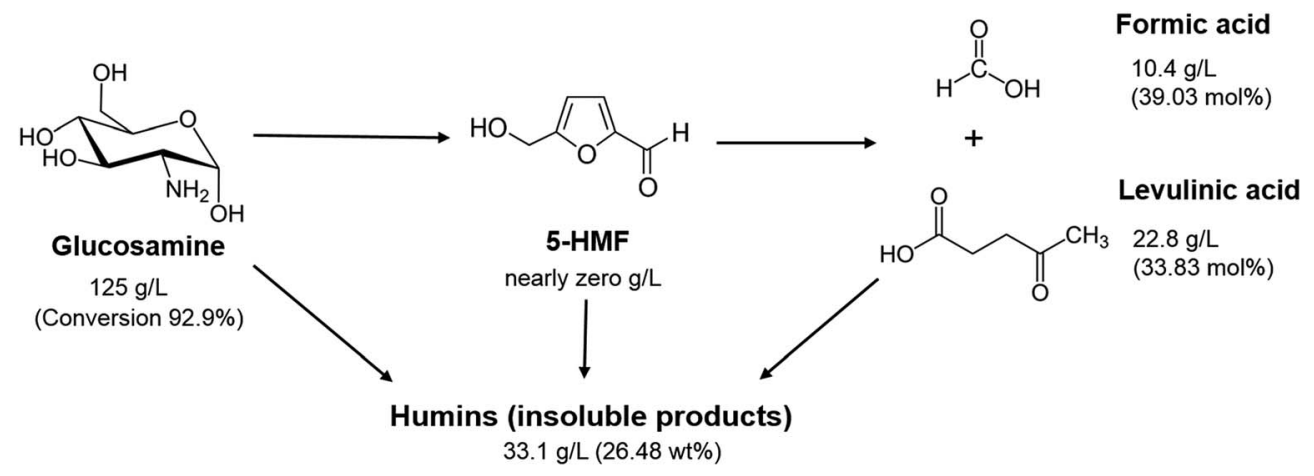

Fig. 6 Mass balance of formation of levulinic acid, formic acid and humins from glucosamine with sulfamic acid. 
Table 1 Comparison of LA production from chitosan and glucosamine

\begin{tabular}{llcl}
\hline Materials & Reaction conditions & LA & References \\
\hline Chitosan & $\mathrm{MI}^{a}, 200{ }^{\circ} \mathrm{C}, 0.24 \mathrm{mmol} \mathrm{SnCl}$ & $\cdot 5 \mathrm{H}_{2} \mathrm{O}, 30 \mathrm{~min}, 100 \mathrm{mg}$ chitosan with $4 \mathrm{~mL}$ water & $23.9 \mathrm{wt} \%$ \\
Glucosamine & $\mathrm{MI}^{a}, 200{ }^{\circ} \mathrm{C}, 0.26 \mathrm{mmol} \mathrm{SnCl}$ & $\cdot 5 \mathrm{H}_{2} \mathrm{O}, 100 \mathrm{mg}$ glucosamine and $20 \mathrm{~mL}$ water & 9 \\
Glucosamine & $\mathrm{CH}^{b}, 188^{\circ} \mathrm{C}, 4 \mathrm{wt} \%$ sulfuric acid, $49 \mathrm{~min}, 120 \mathrm{~g} \mathrm{~L}^{-1}$ glucosamine & 9 \\
Glucosamine & $\mathrm{CH}^{b}, 200{ }^{\circ} \mathrm{C}, 125 \mathrm{~g} \mathrm{~L}^{-1}$ glucosamine, $0.3 \mathrm{M} \mathrm{sulfamic}$ acid and $15 \mathrm{~min}$ & $25.3 \mathrm{wt} \%$ & 11 \\
\end{tabular}

${ }^{a}$ MI: microwave irradiation. ${ }^{b} \mathrm{CH}$ : conventional heating.

\section{Effect of combined severity factor}

The effect of the sulfamic acid-catalyzed hydrothermal reaction on the 5-HMF and LA yield was evaluated using the CSF. Fig. 7 shows the relationships of CSF value and 5-HMF or LA yield in the sulfamic acid-catalyzed hydrothermal conversion of glucosamine. In the 5-HMF case (Fig. 6(A)), increasing CSF values decreased the 5-HMF yield. This is well fitted to the regression pattern of exponential decay $\left(f=y_{0}+a \exp (-b x) ; y_{0}=\right.$ $-0.350, a=244.637, b=1.971)$, with regression value $\left(R^{2}\right)$ of 0.811 (Adj. $R^{2}=0.805$ ). The highest 5 -HMF yield was $6.36 \%$ at CSF 1.77. Over CSF 3 , the 5-HMF yields were nearly zero. In the case of LA production, increasing CSF value sharply linearly increased the LA yield until CSF 3, and then with further increasing CSF value, steadily maintained it. The highest LA yield is obtained near the CSF 3 range. It is well fitted to the regression pattern of the sigmoidal model $(f=a /(1+\exp (-(x-$ $\left.\left.x_{0}\right) / b\right)$ ); $\left.a=33.196, x_{0}=2.122, b=0.233\right)$ with high regression value $\left(R^{2}\right)$ of $0.934\left(\mathrm{Adj} . R^{2}=0.932\right)$. This indicates that in the sulfamic acid-catalyzed hydrothermal reaction of glucosamine, a higher CSF, which is a harsh reaction condition, can significantly convert it to LA. ${ }^{11}$

\section{Comparison of LA production from glucosamine and chitosan}

Table 1 shows the results of comparable work on LA production from chitosan and glucosamine. In Omari et al., ${ }^{9}$ chitosan and glucosamine at $200{ }^{\circ} \mathrm{C}$ with $\mathrm{SnCl}_{4} \cdot 5 \mathrm{H}_{2} \mathrm{O}$ as catalyst under microwave irradiation were converted into LA yields of $23.9 \mathrm{wt} \%$ and $32.0 \mathrm{wt} \%$, respectively. Jeong ${ }^{11}$ reported that the sulfuric acid-catalyzed hydrothermal reaction, under the conditions of $188^{\circ} \mathrm{C}, 4 \mathrm{wt} \%$ sulfuric acid, $120 \mathrm{~g} \mathrm{~L}^{-1}$ glucosamine, and $49 \mathrm{~min}$, produced $25.3 \mathrm{wt} \%$ of LA yield. Comparably, in this work, in the sulfamic acid-catalyzed hydrothermal reaction, the conditions of $200{ }^{\circ} \mathrm{C}, 125 \mathrm{~g} \mathrm{~L} \mathrm{~L}^{-1}$ glucosamine, $0.3 \mathrm{M}$ sulfamic acid, and 15 min produced a $33.76 \pm 0.19 \mathrm{~mol} \%$ LA yield. In the above reports, the LA yield varies with the reaction conditions and substrate.

\section{Conclusions}

In this study, glucosamine, which is a monomer of chitosan, and sulfamic acid, which has dual active sites, were employed as substrate and catalyst, respectively, to produce the bio-based platform chemicals LA and 5-HMF. By optimization of LA yield from glucosamine in the sulfamic acid-catalyzed hydrothermal reaction, the conditions of $200{ }^{\circ} \mathrm{C}, 125 \mathrm{~g} \mathrm{~L}^{-1}$ glucosamine, $0.3 \mathrm{M}$ sulfamic acid, and 15 min achieved a 33.76 $\pm 0.19 \mathrm{~mol} \%$ LA yield. The same conditions produced only $0.14 \mathrm{~mol} \%$ of 5 -HMF yield. These results show the potential and availability in the field of biofuels and materials synthesis of glucosamine as a bioresource, and sulfamic acid as catalyst.

\section{Conflicts of interest}

There are no conflicts to declare.

\section{Acknowledgements}

This research was supported by Basic Science Research Program through the National Research Foundation of Korea (NRF) funded by the Ministry of Education (NRF-2015R1D1A3A01015882).

\section{References}

1 S. K. Hoekman, Renewable Energy, 2009, 34, 14-22.

2 D. J. Hayes, S. Fitzpatrick, M. H. B. Hayes and J. R. H. Ross, in Biorefineries - Industrial Processes and Products, WILEY-VCH Verlag GmbH, Weinheim, Germany, 2006, pp. 139-164.

3 J. J. Bozell and G. R. Petersen, Green Chem., 2010, 12, 539554.

4 O. M. Kwon, D. H. Kim, S. K. Kim and G. T. Jeong, Algal Res., 2016, 13, 293-297.

5 T. H. Nguyen, C. H. Ra, Y. I. Sunwoo, G. T. Jeong and S. K. Kim, J. Microbiol. Biotechnol., 2016, 26, 1264-1271.

6 A. Morone, M. Apte and R. A. Pandey, Renewable Sustainable Energy Rev., 2015, 51, 548-565.

7 A. Mukherjee, M. J. Dumont and V. Raghavan, Biomass Bioenergy, 2015, 72, 143-183.

8 S. B. Lee and G. T. Jeong, Appl. Biochem. Biotechnol., 2015, 176, 1151-1161.

9 K. W. Omari, J. E. Besaw and F. M. Kerton, Green Chem., 2012, 14, 1480-1487.

10 Y. Wang, C. M. Pederson, T. Deng, Y. Qiao and X. Hou, Bioresour. Technol., 2013, 143, 384-390.

11 G. T. Jeong, Ind. Crops Prod., 2014, 62, 77-83.

12 S. Yu, H. Zang, S. Chen, Y. Jiang, B. Yan and B. Cheng, Polym. Degrad. Stabil., 2016, 134, 105-114.

13 H. Zang, S. Yu, P. Yu, H. Ding, Y. Du, Y. Yang and Y. Zhang, Carbohydr. Res., 2017, 442, 1-8.

14 D. H. Kim, S. B. Lee, S. K. Kim, D. H. Park and G. T. Jeong, BioEnergy Res., 2016, 9, 1155-1166. 
15 G. T. Jeong, C. H. Ra, Y. K. Hong, J. K. Kim, I. S. Kong, S. K. Kim and D. H. Park, Bioprocess Biosyst. Eng., 2015, 38, 207-217.

16 S. K. Kim, Chitin, chitosan, oligosaccharides and their derivatives : Biological activities and applications, CRC Press, New York, 2011.

17 S. Tokura and H. Tamura, in Comprehensive Glycoscience, Elsevier Ltd., Amsterdam, 2007, vol. 2, pp. 449-475.

18 R. A. A. Muzzarelli, Carbohydr. Polym., 1993, 20, 7-16.

19 A. Percot, C. Viton and A. Domard, Biomacromolecules, 2003, 4, 8-18.

20 M. N. V. Ravikumar, React. Funct. Polym., 2001, 46, 1-27.

21 P. C. Bersch, B. Nies and A. Liebendorfer, J. Mater. Sci.: Mater. Med., 1995, 6, 231-240.

22 S. K. Kim and N. Rajapakse, Carbohydr. Polym., 2005, 62, 357-368.

23 M. G. Montes D'Oca, R. M. Soares, R. R. de Moura and V. de Freitas Granjao, Fuel, 2012, 97, 884-886.

24 J. Sun, X. Yuan, Y. Shen, Y. Yi, B. Wang, F. Xu and R. Sun, Ind. Crops Prod., 2015, 70, 266-271.

25 G. A. Benson and W. J. Spillane, Chem. Rev., 1980, 80, 151186.

26 A. Kamal, K. S. Babu, S. M. Ali Hussaini, P. S. Srikanth, M. Balakrishna and A. Alarifi, Tetrahedron Lett., 2015, 56, 4619-4622.

27 M. Pedersen and A. S. Meyer, New Biotechnol., 2010, 27, 739750.

28 B. F. M. Kuster, Starch, 1990, 42, 314-321.
29 N. Ya'aini, N. A. S. Amin and S. Endud, Microporous Mesoporous Mater., 2013, 171, 14-23.

30 R. J. Putten, J. C. Waal, E. Jong, C. B. Rasrendra, H. J. Heeres and J. G. Vries, Chem. Rev., 2013, 113, 1499-1597.

31 H. Rasmussen, H. R. Sorensen and A. S. Meyer, Carbohydr. Res., 2014, 385, 45-57.

32 J. Zhang and N. Yan, Green Chem., 2016, 18, 5050-5058.

33 Y. Yang, C. W. Hu and M. M. Abu-Omar, Bioresour. Technol., 2012, 116, 190-194.

34 S. De, S. Dutt and B. Saha, Green Chem., 2011, 13, 2859-2868.

35 L. Audrieth, M. Sveda, H. Sisler and M. J. Butler, Chem. Rev., 1940, 26, 49-94.

36 Y. Jiang, H. Zhang, S. Han, B. Yan, S. Yu and B. Cheng, RSC $A d v .$, 2016, 6, 103774-103781.

37 W. Weiqi and W. Shubin, Chem. Eng. J., 2017, 307, 389-398.

38 H. Xin, T. Zhang, W. Li, M. Su, S. Li, Q. Shao and L. Ma, RSC Adv., 2017, 7, 41546-41551.

39 C. Wang, L. Zhang, T. Zhou, J. Chen and F. Xu, Sci. Rep., 2017, DOI: $10.1038 /$ srep40908.

40 X. Zhang, P. Murria, Y. Jiang, W. Xiao, H. I. Kenttamaa, M. A. Abu-Omar and N. S. Mosier, Green Chem., 2016, 18, 5219-5229.

41 T. C. Acharjee and Y. Y. Lee, Environ. Prog. Sustainable Energy, 2017, DOI: 10.1002/ep.12659.

42 X. F. Sun, R. Sun, P. Fowler and M. S. Baird, J. Agric. Food Chem., 2005, 53, 860-870.

43 M. Kačuráková, P. Capek, V. Sasinková, N. Wellner and A. Ebringerová, Carbohydr. Polym., 2000, 43, 195-203. 